\title{
Material Selection for Axial Magnetization of a Gyromagnetic NLTL for Space Applications
}

\author{
André F. Teixeira ${ }^{1}$, Fernanda S. Yamasaki ${ }^{1}{ }^{\circledR}$, José O. Rossi ${ }^{1}{ }^{\circledR}$, Joaquim J. Barroso ${ }^{2}{ }^{\circledR}$, Ana Flávia

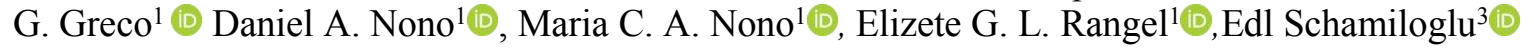 \\ ${ }^{1}$ National Institute for Space Research (INPE), 12227-010, São José dos Campos, SP, Brazil, \\ andre-zth@hotmail.com,fernandayamasaki@hotmail.com, jose.rossi@inpe.br, \\ anaflaviaguedesgreco@gmail.com,daniel.a.nono@hotmail.com,maria.nono@inpe.br,elizete.rangel@inpe.br \\ ${ }^{2}$ Technological Institute of Aeronautics (ITA), 12228-090 São José dos Campos-SP, Brazil, barroso@ita.br \\ ${ }^{3}$ University of New Mexico (UNM), 87131, Albuquerque, NM, USA, edls@unm.edu
}

\begin{abstract}
Nonlinear Transmission Lines (NLTLs) are a new technique for radio frequency (RF) generation. A loaded ferrite NLTL, known as a gyromagnetic line, uses a solenoid to provide an external magnetic bias. In space applications, specifically in satellites, the replacement of these solenoids by permanent magnets is desirable, eliminating the need for a DC current source, and reducing the weight and the effective cost of the launch. This work investigated and selected permanent magnets for this application, and then computationally modeled the magnet assembly to analyze the resulting magnetic field generated and obtained a uniform field region to meet the NLTL operating specifications. For this, we employed selection charts for the proper choice of material to use in an arrangement of magnets simulated by the electromagnetic software CST Microwave Studio. Magnetic fields with uniformity variations of less than $6 \%$ and $23 \%$ in regions extended over 18.5 $\mathrm{cm}$ and $58.8 \mathrm{~cm}$, corresponding to line lengths of $26.6 \mathrm{~cm}$ and 68.0 $\mathrm{cm}$, respectively, were achieved in simulations.
\end{abstract}

Index Terms - Gyromagnetic nonlinear transmission lines, material selection, NLTLs, permanent magnet.

\section{INTRODUCTION}

Nonlinear Transmission Lines (NLTLs) are devices used to generate radiofrequency (RF) for the replacement of electronic vacuum tubes and their thermionic filaments [1]. This technology represents a new alternative to generate RF without the use of an electron beam or need for a vacuum. They are one of two types: (1) a dispersive network of inductor-capacitor (LC) cells in the VHF band (5-400 $\mathrm{MHz}$ ) [2], [3] where at least one component must be nonlinear ( $\mathrm{L}$ or C) and (2) a continuous coaxial line, called a gyromagnetic line, operating in $\mathrm{L}$ band $(1-2 \mathrm{GHz})$ [4]. The latter one is the focus of the study in this work.

Gyromagnetic NLTLs can generate high power (hundreds of MW) [5], [6], enabling applications in pulsed radar satellite (SAR) systems. There is great interest in compacting these lines for use as highpower microwave (HPM) sources, mounted on small defense platforms (boats and trucks) with operational applications to neutralize enemy electronic systems [1], so-called electronic attack. In conventional gyromagnetic lines, the magnetic propagation medium is formed by magnetic ferrite 
Journal of Microwaves, Optoelectronics and Electromagnetic Applications, Vol. 20, No. 3, September 2021 DOI: http://dx.doi.org/10.1590/2179-10742021v20i3254769

cores immersed in a constant external axial magnetic field. Figure 1 illustrates the generation of RF in a coaxial gyromagnetic NLTL from an applied rectangular input voltage pulse. The RF oscillations are generated by the precession of the ferrites' magnetic moments when excited by the azimuthal magnetic field created by the pulsed current that interacts with the external axial magnetic field generated using a solenoid.

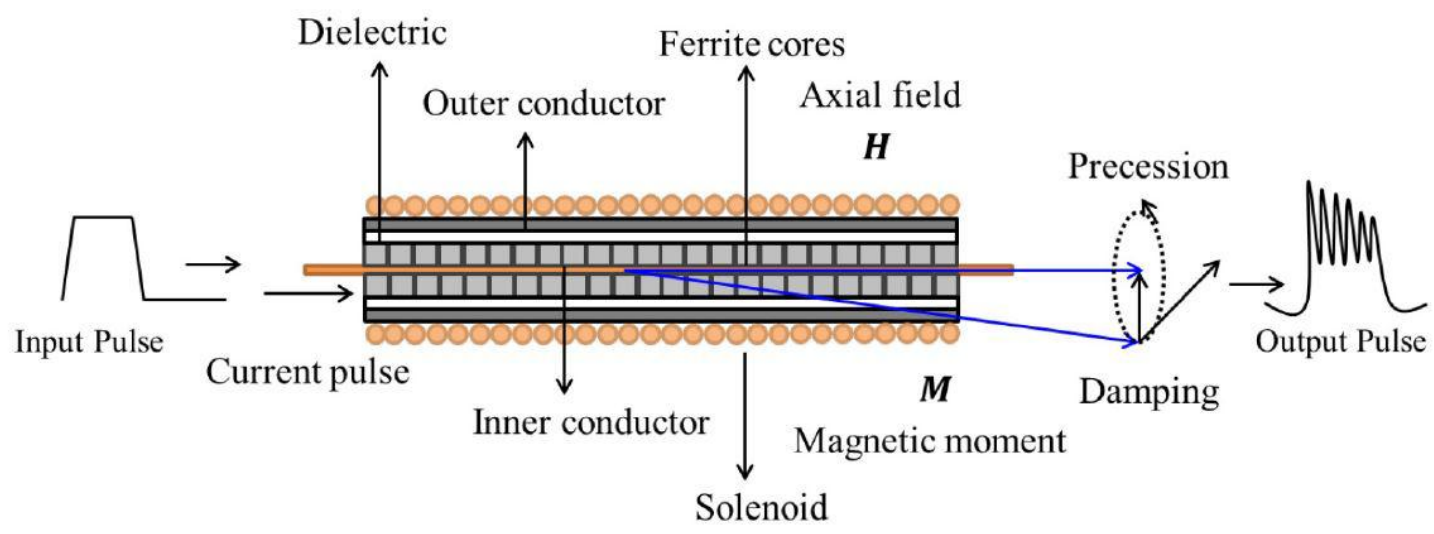

Fig. 1. Effect of magnetic precession on a gyromagnetic line [1].

The effect of damped gyromagnetic precession is described by the Landau-Lifshitz-Gilbert (LLG) equation [7], which in CGS units is given by

$$
\frac{\partial \vec{M}}{\partial t}=\gamma \mu_{0} \vec{M} \times \vec{H}-\frac{\alpha}{M_{s}} \vec{M} \times \frac{\partial \vec{M}}{\partial t}
$$

This equation describes the magnetization dynamics that occurs in ferrites [8], [9]. In (1), $\vec{M}$ is the magnetization vector, $\vec{H}$ is the axial magnetic field vector, $\overrightarrow{M_{s}}$ is the saturation magnetization, $\gamma$ is the electron gyromagnetic relationship $\left(1.760 \times 10^{11} \mathrm{rad} \cdot \mathrm{s}^{-1} \cdot \mathrm{T}^{-1}\right), \alpha$ is the precession damping constant dependent on the material and has typical values of $0.001-0.1$, and $\mu_{0}$ is the vacuum magnetic permeability. The external polarization $(\vec{H})$ traditionally originates from a solenoid with the NLTL positioned inside it. Figure 2 shows the experimental arrangement [10] and Fig. 3 presents the corresponding block diagram.

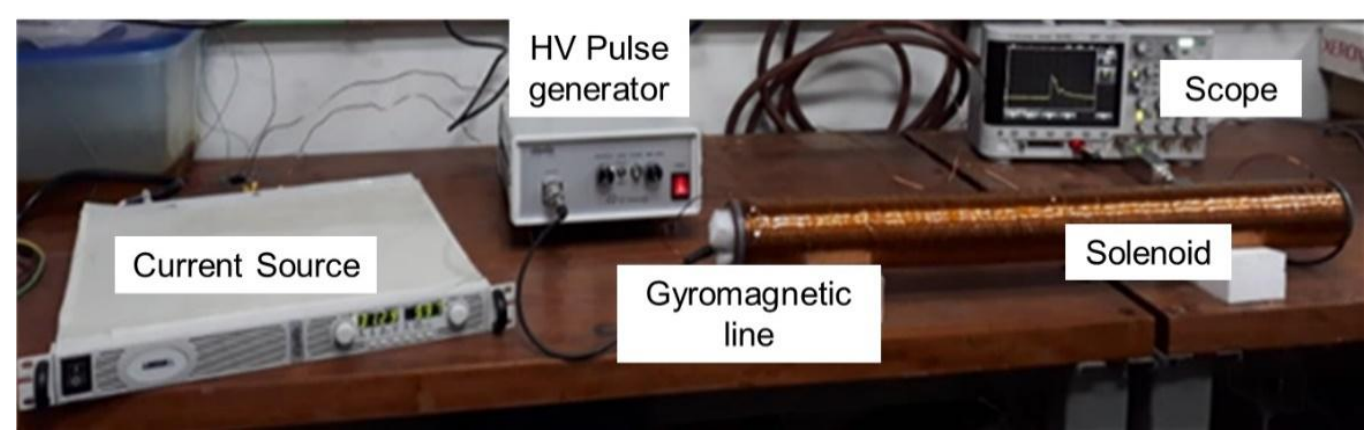

Fig. 2. System for testing a gyromagnetic line using a solenoid as an external axial magnetic field source [10].

The output pulse of the NLTL is measured by coupling an $80 \mathrm{~dB}$ attenuator to the $50 \Omega$ channel 
Journal of Microwaves, Optoelectronics and Electromagnetic Applications, Vol. 20, No. 3, September 2021

DOI: http://dx.doi.org/10.1590/2179-10742021v20i3254769

input impedance of a digital oscilloscope. This system is used to test the NLTL before removing the DC level of the output signal through a high-frequency pass filter to irradiate with an antenna.

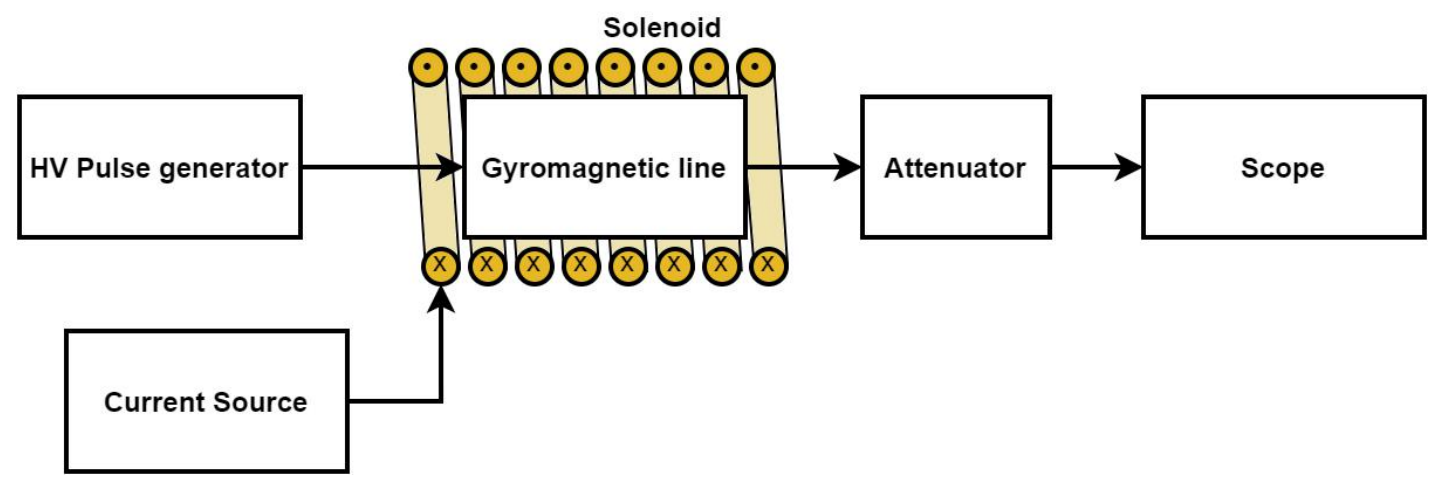

Fig. 3. Block diagram of the experiment used to test the NLTL.

According to analytical results and experimental data, an acceptable range of axial magnetic fields extends from $20 \mathrm{kA} / \mathrm{m}$ to $40 \mathrm{kA} / \mathrm{m}$ [8], [11]. The axial magnetic field is combined with the generated azimuthal one to saturate the ferrites. In this way, it produces the precession movement of the electron spins (gyromagnetic effect). However, with an axial magnetic field greater than $40 \mathrm{kA} / \mathrm{m}$, the precession oscillations vanish [11]. Although the use of solenoids in these devices is typical, Bragg [9] has obtained promising results for replacing them with permanent magnets, despite not achieving a sufficiently uniform magnetic field. Also, [12] and [13] give studies on magnetic field uniformity using permanent magnets.

This research deals with permanent magnets that best meet the requirements (density, operating temperature, and magnetic field intensity) for compact space-based gyromagnetic NLTLs. With the aid of the electromagnetic CST Studio Suite software, a configuration that allows for a uniform magnetic field for axial polarization of the gyromagnetic line using permanent magnets is presented. For this, we investigated the material selection process for space applications, selecting the most suitable material for the application. The main idea here is the weight reduction of the NLTL device in space applications by using an arrangement of permanent magnet blocks to replace solenoid and DC current source. This technique provides more than $80 \%$ weight reduction as blocks of neodymium magnets are lighter than a solenoid and its DC supply. Another scientific contribution of this work is to propose a cylindrical structure using an arrangement of blocks on polygonal contours [14] for producing a uniform magnetic field to replace the solenoid in gyromagnetic NLTLs. The novel aspect herein is that this technique has been used only for applications in medical equipment of nuclear magnetic resonance imaging.

\section{MATERIAL SELECTION FOR SPACE USE}

Several environmental requirements restrict the selection of materials for space applications. The materials to be selected, among other properties, must be light to minimize the payload mass. The reduced weight of a space vehicle or satellite directly affects the amount of fuel used, providing 
Journal of Microwaves, Optoelectronics and Electromagnetic Applications, Vol. 20, No. 3, September 2021

DOI: http://dx.doi.org/10.1590/2179-10742021v20i3254769

enormous economic and ecological benefits [15]. The main objectives for the development of materials in the aerospace industry are to reduce weight and increase resilience to abrupt temperature variations. Thus, selecting an ideal material from a wide variety of materials available for a specific application is not a trivial task. There are more than 21 different aspects and phenomena in space that can affect the physical properties of materials and, consequently, the performance of their associated devices [16]. The influence of the space environment on materials depends mainly on the type of mission, the solar period in which the mission will take place, as well as on the orbit in which a satellite orbits.

Among the various methods of material selection found in the literature, what stands out is the method of Ashby [16], which relates two specific properties of materials through charts. As the performance of a component usually depends on a combination of properties, the Ashby method arises from the idea of plotting one property in the function of the other, mapping them through regions called islands, occupied by each material. A function, called the merit index, is determined to describe a dividing line between possible and non-possible materials. A secondary limitation can be used, which allows one to further detail the area of choice of materials. The resulting graphics, called selection charts, are useful in several ways. They encompass a large body of information in a more accessible, compact form, and reveal correlations between the properties of materials [16].

Considering all of the factors of the space environment at once would make the problem very complicated. For simplicity, in this paper, two factors of the spatial environment are examined: density (related to mass) and temperature in the operating environment. As seen previously, mass directly reflects the cost of launching any artifact into space, and in the case of satellites, the temperature varies drastically according to its orbital movement, passing through eclipse regions (without looking at the sun) and illuminated regions (exposed to the sun). The functional requirement of the axial magnetic field (necessary for the external polarization of the gyromagnetic NLTL) must also be considered. This was obtained experimentally and analytically in previous works [8] and [11].

Here we consider that the NLTL is embedded internally on a satellite. Manufacturers use different technologies to construct their satellites, with it being common to vary the operating temperature limits from one satellite to another [17] according to the thermal balance of each system.

The gyromagnetic line must operate under the thermal control of the satellite system within the temperature range of $0^{\circ} \mathrm{C}$ to $100^{\circ} \mathrm{C}$, thus ensuring an operating safety margin to enable space applications [18]. It should be noted that Bragg reports from experiments that these lines operate with satisfactory performance between $-10{ }^{\circ} \mathrm{C}$ and $130{ }^{\circ} \mathrm{C}[19,20]$.

\section{PERMANENT MAGNETS}

Permanent magnets have a permanent magnetization since they store magnetic energy. However, they can lose their magnetization if exposed to high temperatures (above the Curie temperature) since mechanical stresses alter the atomic distribution of the crystals or high external magnetic fields 
Journal of Microwaves, Optoelectronics and Electromagnetic Applications, Vol. 20, No. 3, September 2021

DOI: http://dx.doi.org/10.1590/2179-10742021v20i3254769

capable of demagnetizing the material.

There are different types of permanent magnets, depending on the geometry, properties, and type of material. The most popular materials for permanent magnets are [21]: Alnico alloys (where their main components are iron, cobalt, nickel and aluminum), ceramic magnets (ferrites), samarium-cobalt (Sm$\mathrm{Co}$ ), neodymium-iron-boron (Nd-Fe-B), and iron-chromium-cobalt (Fe-Cr-Co). Several geometries can be implemented using the shape of disks, rings, cylinders, blocks, bars, and cubes. Each manufacturer can provide custom geometries.

The main magnetic characteristics for the selection of magnets are obtained through their demagnetization curves $(B-H)$. Such curves are determined by analyzing the second quadrant of the hysteresis cycle shown in Fig. 4, and indicate how the magnetic flux density $B$ in a magnet varies with the demagnetization field $H$ applied.

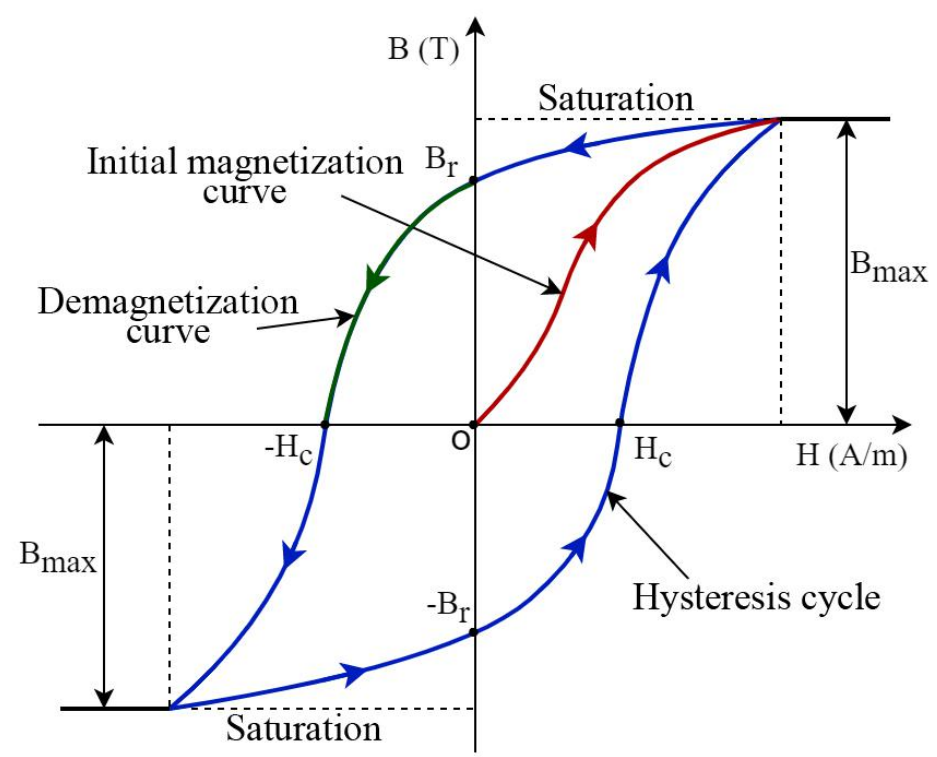

Fig. 4. Hysteresis cycle.

The process of magnetizing a material is carried out by applying an external magnetic field. Initially demagnetized, the material begins to suffer the action of the applied external field $H$. Starting from the origin $\mathrm{O}(0,0)$ in Fig. 4, there is an increase in the magnetization of the material with the applied external field. The material then reaches the saturation level $\left(B_{\max }\right)$. As the intensity of $H$ decreases, the magnetic density flux $B$ of the material decreases, but the flux does not follow the initial magnetization path on the $B-H$ curve as seen in Fig. 4 .

Some permanent magnets have the property of being hardly demagnetized (hard materials), having high coercivity $\left(H_{c}\right)$, which measures the resistance of the magnetic material to the demagnetization. Remanence $\left(B_{r}\right)$ quantifies the magnetism that remains after removing the external magnetic field ( $H$ $=0$ ). The proper choice of a permanent magnet takes into account several factors (mechanical, thermal, magnetic, geometric, etc. ) [21]. In this work, the aspects considered critical for the initial analysis using the selection charts were: (1) remanence $\left(B_{r}\right),(2)$ material density, and (3) maximum operating temperature. 
Journal of Microwaves, Optoelectronics and Electromagnetic Applications, Vol. 20, No. 3, September 2021

DOI: http://dx.doi.org/10.1590/2179-10742021v20i3254769

\section{METHODOLOGY}

The methodology used in this work was divided into four parts:

1. A survey of the properties of permanent magnets was carried out using the website databases of national and international manufacturers to build the selection charts for the appropriate materials.

2. The limits allowed by the design requirements were inserted into the charts to reduce the number of solutions suitable for the design requirements of NLTLs.

3. The set of materials appropriate to the design requirements was selected to choose the suitable materials, considering aspects not described by the charts, such as material resistance and demagnetization curve.

4. The electromagnetic simulations of the arrangement using the selected permanent magnets were performed using the software CST StudioSuite ${ }^{\circledR}$ [22], version 2019, to obtain a flat profile of the magnetic field along the length of the line. For running simulations, the CST Magnetostatic Solver was employed without including Ohmic losses caused by eddy currents.

\section{RESULTS AND DISCUSSION}

\section{A. Generation of the Selection Charts}

After the compilation of 574 magnets for the selection of the material properties database, three selection charts were plotted to map some properties of the magnets. Figures 5, 6, and 7 show the mapped regions for the different materials according to their properties.

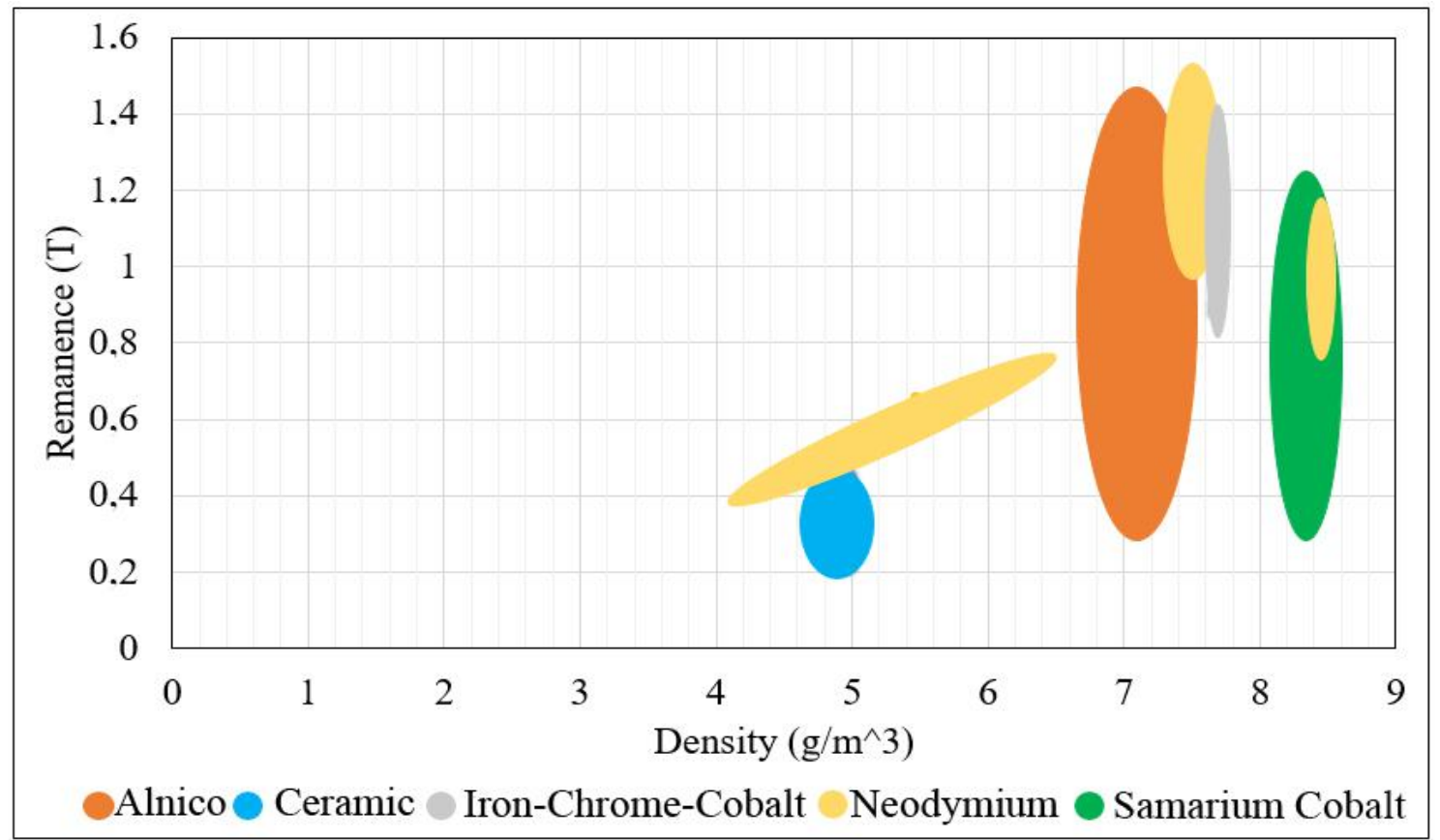

Fig. 5. Selection chart: remanence by density. 


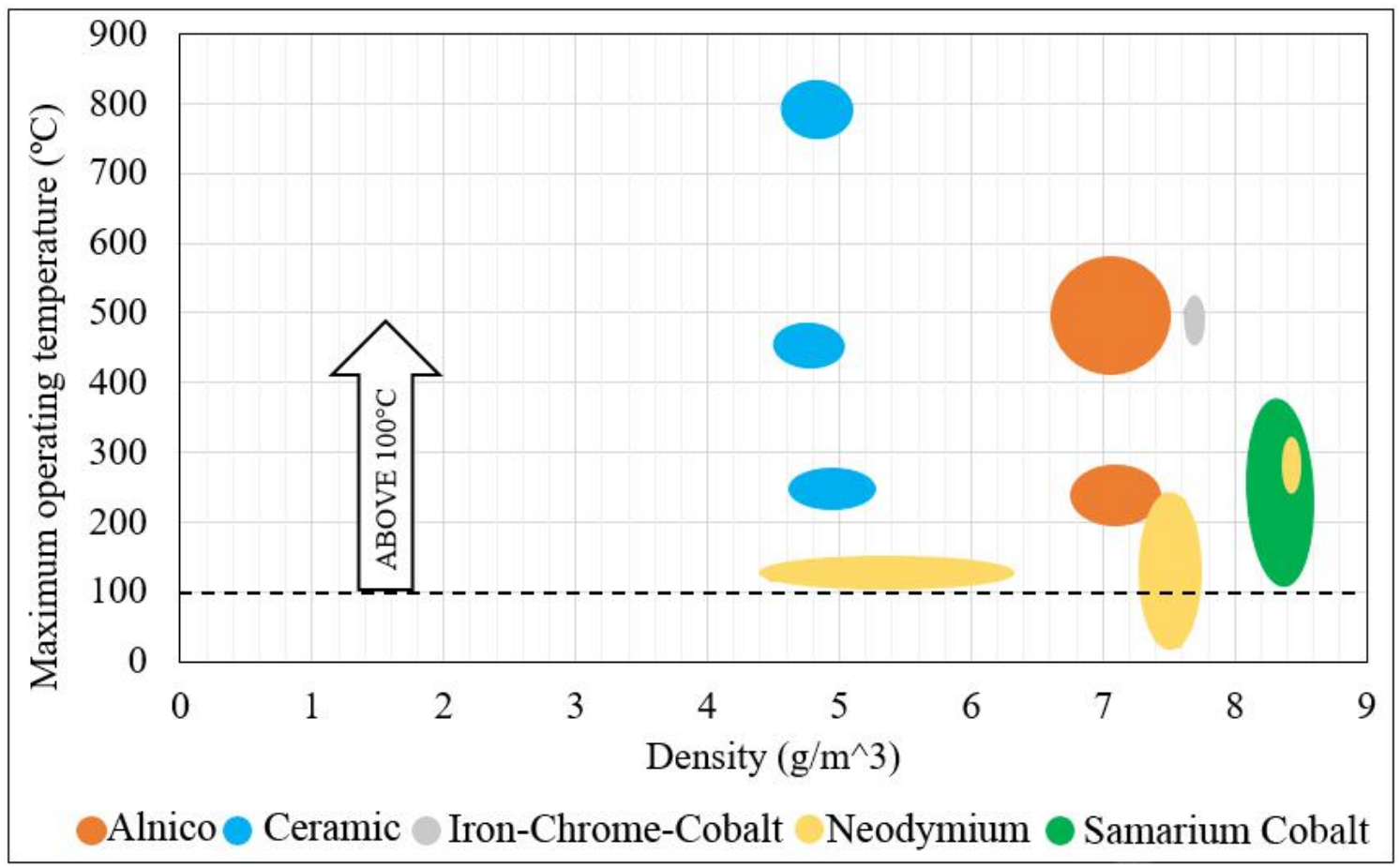

Fig. 6. Selection chart: maximum operating temperature by density.

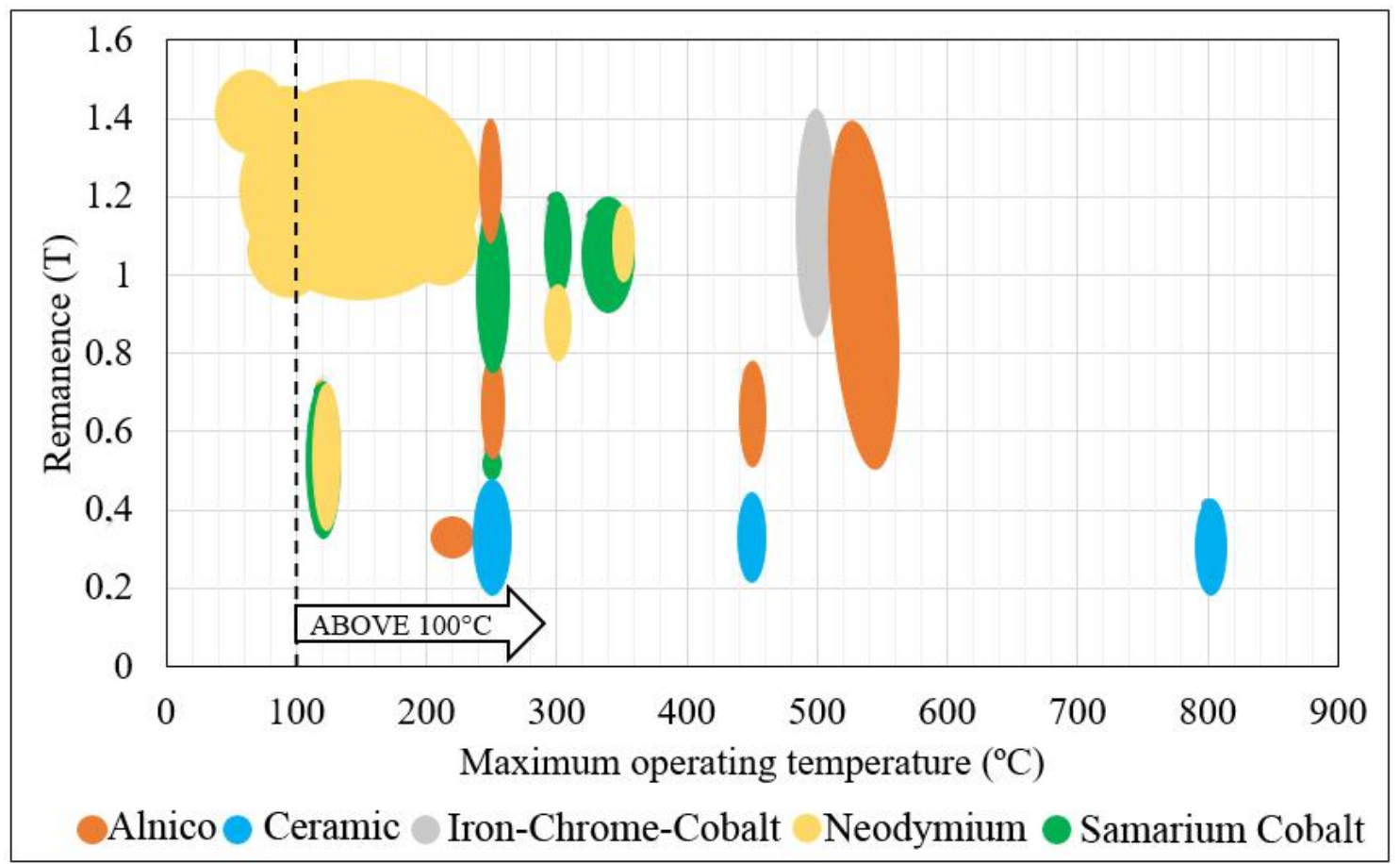

Fig. 7. Selection chart: remanence by maximum operating temperature.

\section{B. Material Selection}

It is observed in the selection charts that ceramic magnets have lower remanence compared to the others. In spite of their lower density, ceramic magnets can operate at high temperatures. It should be noted that a restricted range of remanence prevents the application of ceramic magnets in devices that require an intense magnetic field.

Samarium-cobalt magnets have higher densities and are in the intermediate range of maximum 
Journal of Microwaves, Optoelectronics and Electromagnetic Applications, Vol. 20, No. 3, September 2021

DOI: http://dx.doi.org/10.1590/2179-10742021v20i3254769

operating temperature compared to other materials. They have a good variation in remanence and are considered resistant to corrosion. The cost of this type of magnet is generally higher than that of neodymium magnets. All magnets are brittle, but the Sm-Co magnet is the most fragile and brittle.

Iron-chromium-cobalt magnets, on the other hand, do not have a wide range of remanence and are reasonably malleable. As it is a semi-hard material, it is more easily demagnetized when exposed to external fields; however, these magnets do not need any anti-corrosion coating.

Alnico and ceramic magnets are the best choices for extremely high-temperature applications, as they have a high maximum operating temperature. Alnico magnets have an intermediate density range when compared to other magnets. They are less dense than Sm-Co and ferrochromium-cobalt magnets, but they are magnets of low coercivity, therefore they are also easily demagnetized.

As shown in the charts, from Fig. 5 to Fig. 7, neodymium magnets have a good range of remanence, high variability in their density, and limited maximum operating temperature. This material must be coated to avoid risks of corrosion; however, they perform better with a smaller volume of the magnet, when compared to other material, being hardly demagnetized.

Therefore, evaluating the advantages and disadvantages of all magnets and using the selection charts, neodymium magnets were chosen to provide the necessary NLTL axial magnetization.

\section{Computer Simulations}

First, we tried a conventional configuration of permanent magnets, where the solenoid is replaced by a series of magnet rings (neodymium) displaced along the axis of the NLTL with a length of $59 \mathrm{~cm}$, as shown in Fig. 8. Gyromagnetic lines are typically 10 to $100 \mathrm{~cm}$ long. NLTL gyromagnetic lines of less than $10 \mathrm{~cm}$ are generally not used as there is a minimum length required to compress the rise time of the input signal and induce the magnetic precession. Above $1 \mathrm{~m}$, longer lines are not used as the losses increase with the length. For the simulations, we set a remanent magnetization value of $1.19 \mathrm{~T}$ for each magnet ring with the following dimensions: internal diameter of $30 \mathrm{~mm}$, external diameter of $51 \mathrm{~mm}$, and thickness of $10 \mathrm{~mm}$. Thirty rings were placed $10 \mathrm{~mm}$ apart from each other covering a length of $59 \mathrm{~cm}$, as seen in Fig. 8. In the simulations, the orientation of the axial magnetic polarization for each ring is set as shown in Fig. 9.

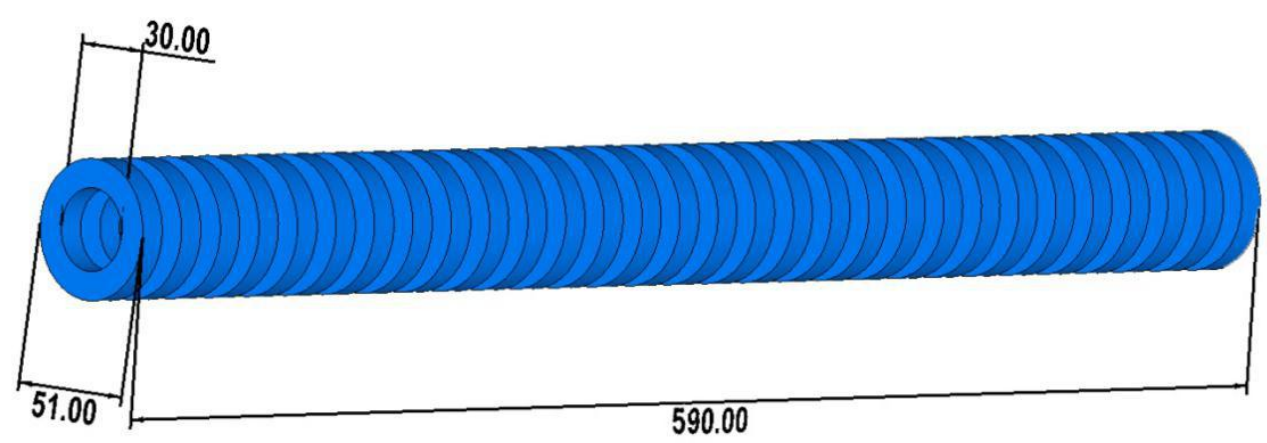

Fig. 8. Arrangement of neodymium rings used in the CST simulations (dimensions in $\mathrm{mm}$ ). 


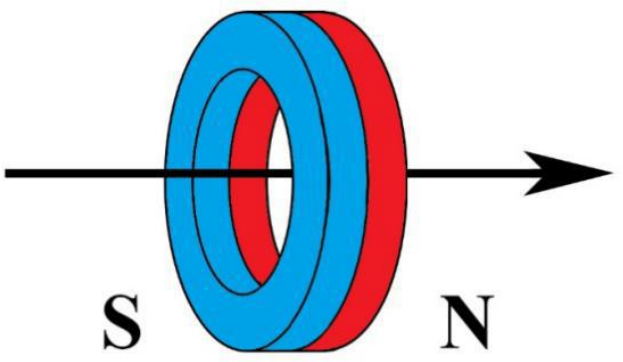

Fig. 9. Illustration of the ring magnetization vector orientation.

Figure 10 shows the corresponding CST magnetic field simulation of the ring arrangement shown in Fig. 8, in which the magnetization vectors invert their orientation, pointing right to left instead of left to right in Fig. 9. The magnetization vector $\vec{M}$ is periodically inverted in each group of five rings. We see, in Fig. 10, that the shape of the curve obtained is not flat as desired along the axial length. However, there are several valleys where the magnetic field drops to almost zero.

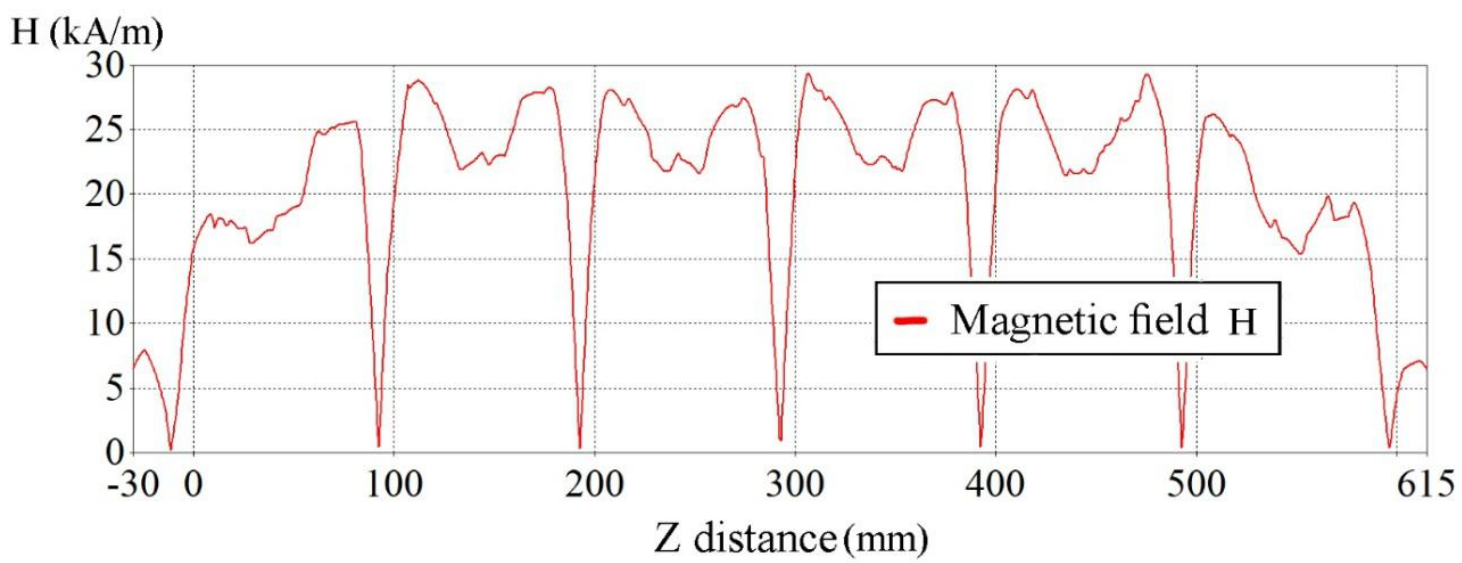

Fig. 10. CST simulation of the magnetic field using the magnet arrangement of Fig. 8.

A different approach needs to be employed as found in [14] with a structure of magnets used in magnetic nuclear resonance equipment to obtain a uniform magnetic field distribution. The patent in [14] only describes the arrangement of magnets and not the methodology to achieve such a result, but they certainly achieved this, running on empirical simulations. First, for the analysis of the generated axial magnetic field using this method, a structure of 8 rings was simulated containing 12 neodymium blocks in each one, as seen in a 3-D view in Fig. 11.

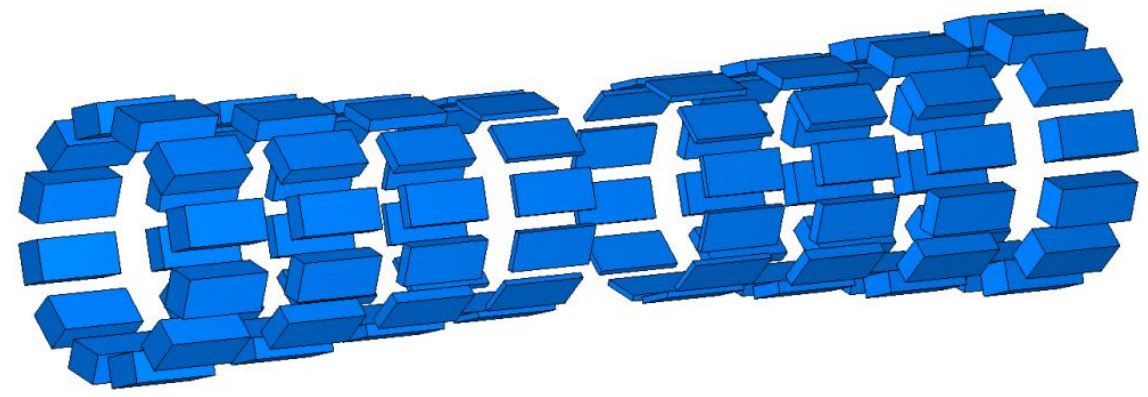

Fig. 11. A 3-D exploded view drawing of the arrangement of rings formed by blocks of neodymium magnets. 
The orientation of the magnetization vector of the simulated blocks is given according to Fig. 12a. The standardization of block measurements can be seen in Fig. 12b.

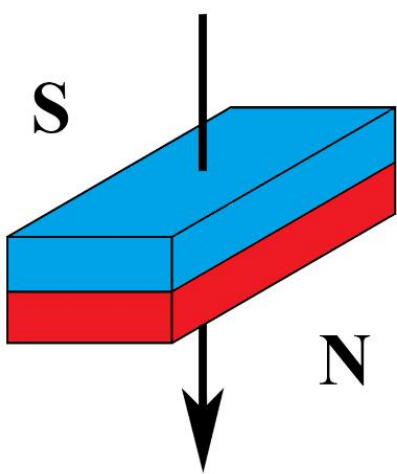

(a)

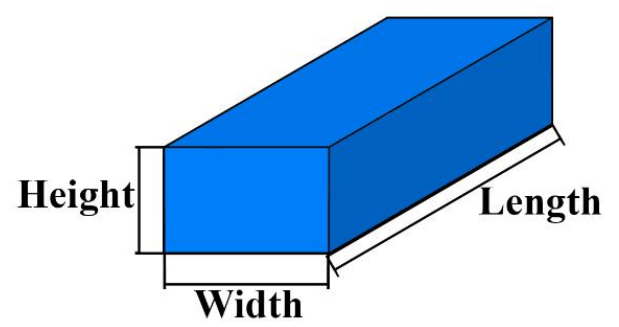

(b)

Fig. 12. a) Illustration of the block magnetization vector orientation and b) Standardization of dimensions used for the simulated block models in this work.

The structure is oriented along the z-axis and its dimensions can be seen in Fig. 13, where the height of the blocks varies from $9.66 \mathrm{~mm}, 6.76 \mathrm{~mm}, 3.86 \mathrm{~mm}$, and $1.93 \mathrm{~mm}$. The width of the blocks is fixed at $12.94 \mathrm{~mm}$ as well as its length at $30 \mathrm{~mm}$. There is a symmetry between the two parts of the device (first 4 rings of blocks and the last 4), the two groups are spaced $26 \mathrm{~mm}$ apart. There is no distance between the rings. The inner diameter of the rings is fixed at $48.30 \mathrm{~mm}$, allowing for the fitting of the NLTL inside the structure.

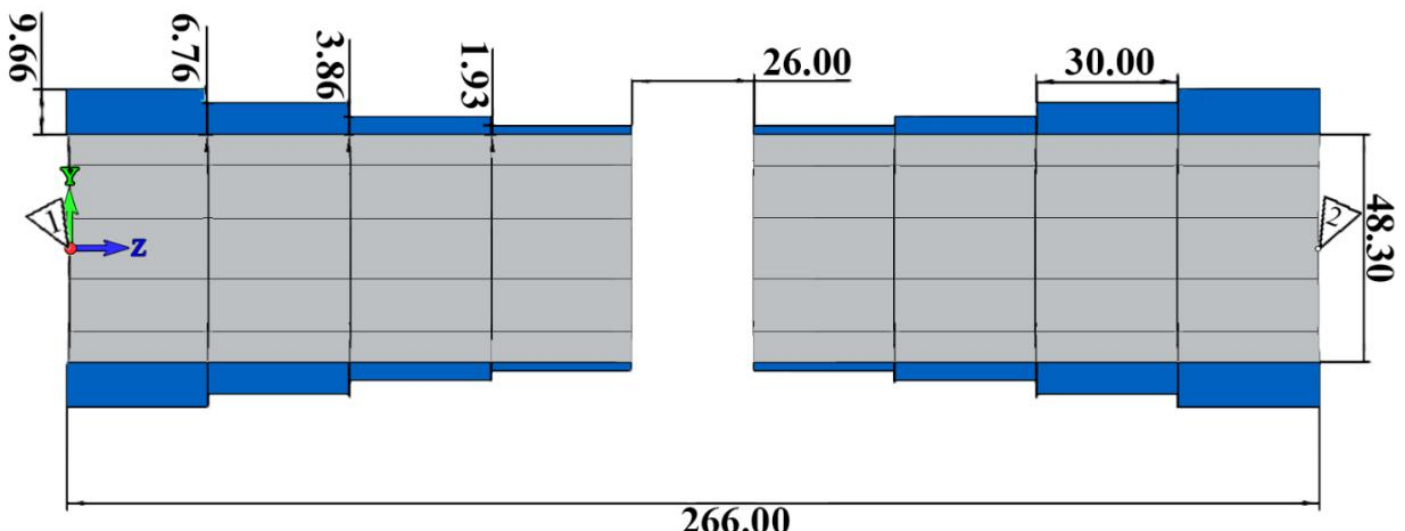

Fig. 13. A 2-D cross-section view of the arrangement of rings. Dimensions in millimeters (thickness, spacing, length, and internal diameter) of the components of the structure.

The orientation of the remanent magnetization vector and its value for each block is shown in Fig. 14. The remanent vector sense for the blocks of each ring formed is the same with the following values from the outside ring to the inside ring at the center for both ring sets: $1.0 \mathrm{~T}, 0.8 \mathrm{~T}, 0.77 \mathrm{~T}$, and 0.73 T. For the first four rings on the left in Fig. 14, the blocks have the same orientation as the magnetization vectors point radially inward, while in the last four rings on the right, the magnetization is radially outward. Thus, using this configuration for the magnets the simulation in Fig. 15 shows a good result, producing a reasonable constant flatness of the axial magnetic field along the $18.5 \mathrm{~cm}$ length of the gyromagnetic line. 


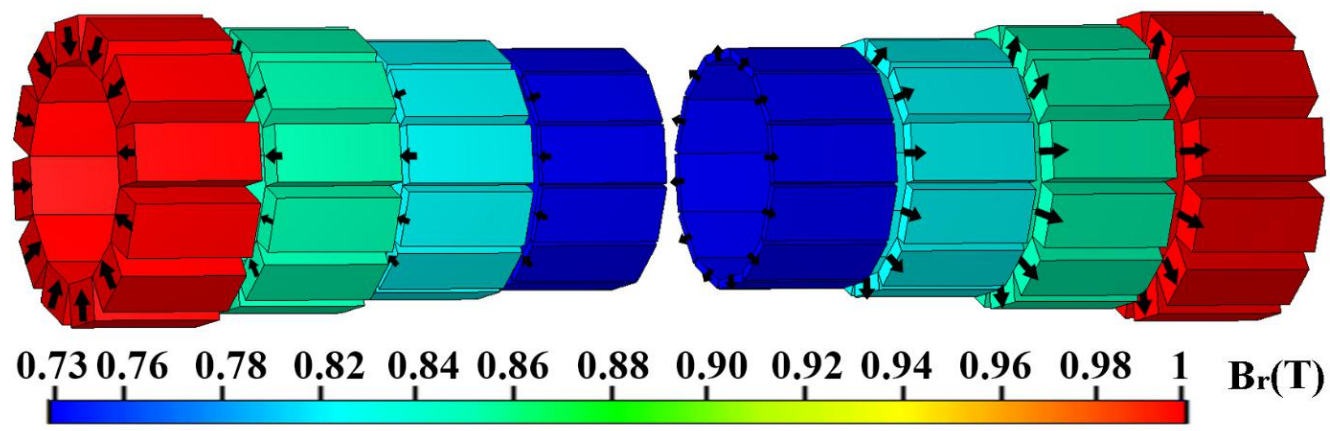

Fig. 14. Remanence flow of the blocks and orientation of the magnetization vectors.

Figure 15 also shows the intensity of the axial magnetic field at the center of the rings, where points 1 and 2 represent the beginning and the end of the ring structure along the z-axis $(26.6 \mathrm{~cm})$, respectively. Besides, it is observed that the magnetic field varies in the range of $28.81 \mathrm{kA} / \mathrm{m}$ to 32.36 $\mathrm{kA} / \mathrm{m}$ along the $18.5 \mathrm{~cm}$ length, enough to provide the required axial magnetic polarization for a 26.6 cm long NLTL.

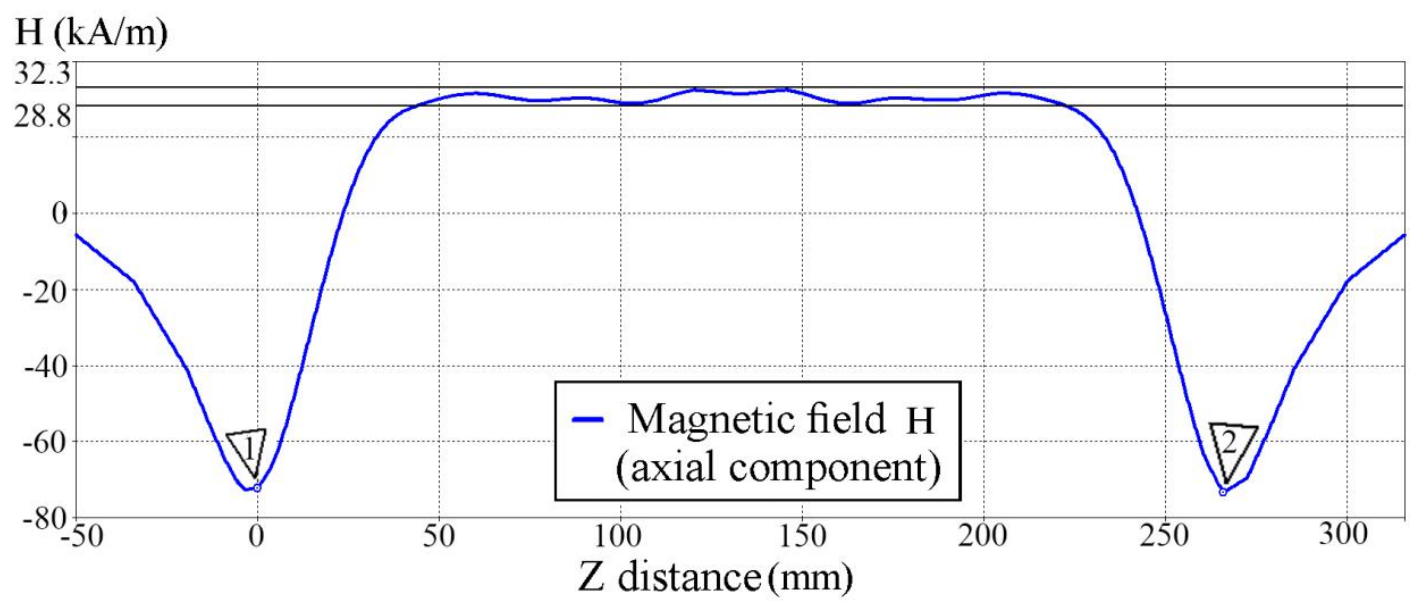

Fig. 15. Magnetic field strength at the center of the rings $(x=0, y=0)$, along the $z$ axis.

Fig. 16 displays the spatial variation of the intensity of the magnetic field, as well as its orientation. There are points where the field lines cancel each other out. This phenomenon occurs due to the magnetic vortex of the reversed vector field lines outside the inner most magnets.

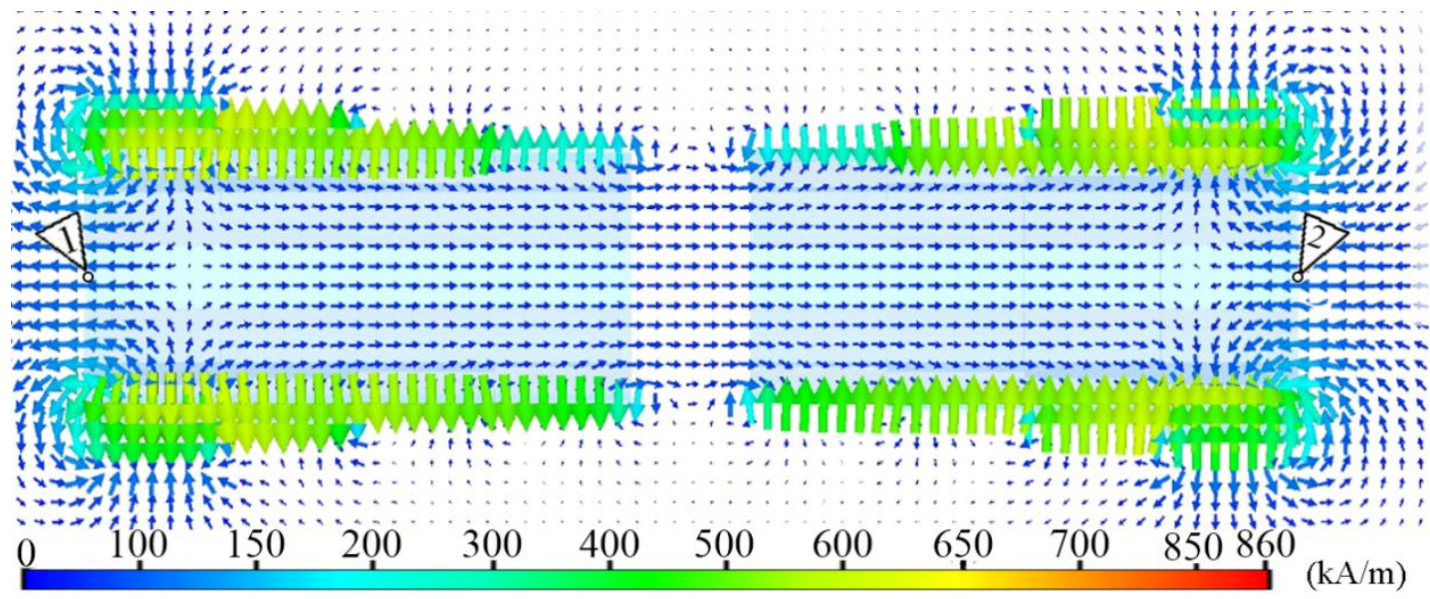

Fig. 16. Magnetic field lines in the cross section of the structure.

Brazilian Microwave and Optoelectronics Society-SBMO Brazilian Society of Electromagnetism-SBMag received 27 Jan 2021; for review 27 Jan 2021; accepted 26 Apr 2021 (c) 2021 SBMO/SBMag (cc) BY 
Journal of Microwaves, Optoelectronics and Electromagnetic Applications, Vol. 20, No. 3, September 2021 DOI: http://dx.doi.org/10.1590/2179-10742021v20i3254769

As an additional example, for the axial magnetization of a gyromagnetic line of approximately 68.0 $\mathrm{cm}$ long, a larger structure was simulated, with 16 sets of neodymium blocks. The dimensions for each neodymium block in the arrangement are $12.9 \mathrm{~mm}$ width, $40.0 \mathrm{~mm}$ length, and $9.7 \mathrm{~mm}, 8.1 \mathrm{~mm}$, $7.0 \mathrm{~mm}, 6.0 \mathrm{~mm}, 5.0 \mathrm{~mm}, 4.0 \mathrm{~mm}, 3.0 \mathrm{~mm}$, and $2.0 \mathrm{~mm}$ height, respectively, following the dimensions standard in Fig. 12. Using the same procedure as the previous arrangement, the first set of 8 rings in this new one has the magnetization orientation contrary to the later set. The remanences of the blocks are: $1.55 \mathrm{~T}, 1.50 \mathrm{~T}, 1.40 \mathrm{~T}, 1.35 \mathrm{~T}, 1.27 \mathrm{~T}, 1.20 \mathrm{~T}, 0.95 \mathrm{~T}$, and $0.80 \mathrm{~T}$, respectively, as seen in Fig. 17.

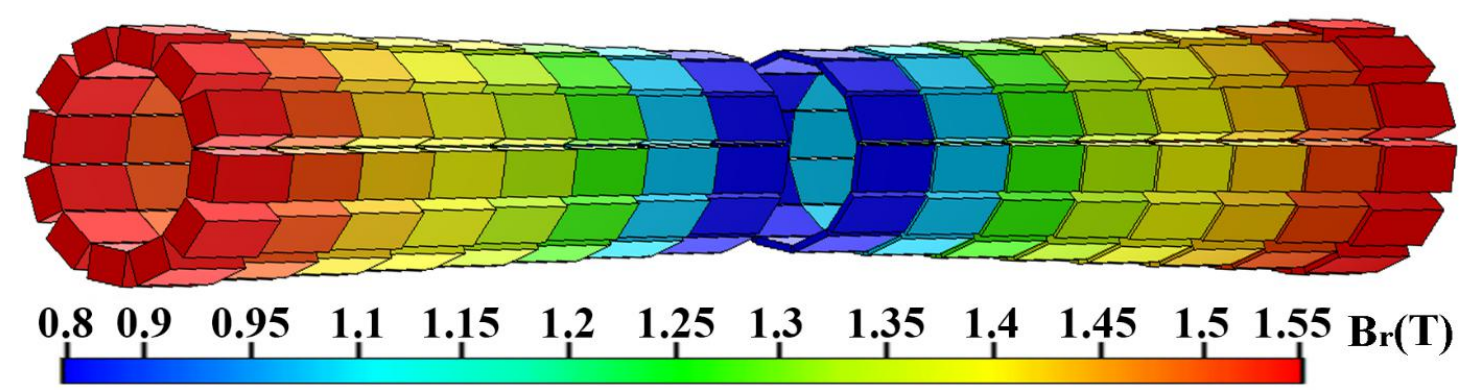

Fig. 17. Remanent flow of the blocks.

Fig. 18 shows the intensity of the axial magnetic field (with $\mathrm{x}=0, \mathrm{y}=0$ ), along the $\mathrm{z}$-axis, where again points 1 and 2 denote the initial and final positions of the block arrangement, respectively. A magnetic field ranging from $22.47 \mathrm{kA} / \mathrm{m}$ to $36.38 \mathrm{kA} / \mathrm{m}$ is obtained, sufficient to allow the functional polarization of a $58.8 \mathrm{~cm}$ extension over a $68-\mathrm{cm}$ NLTL line.

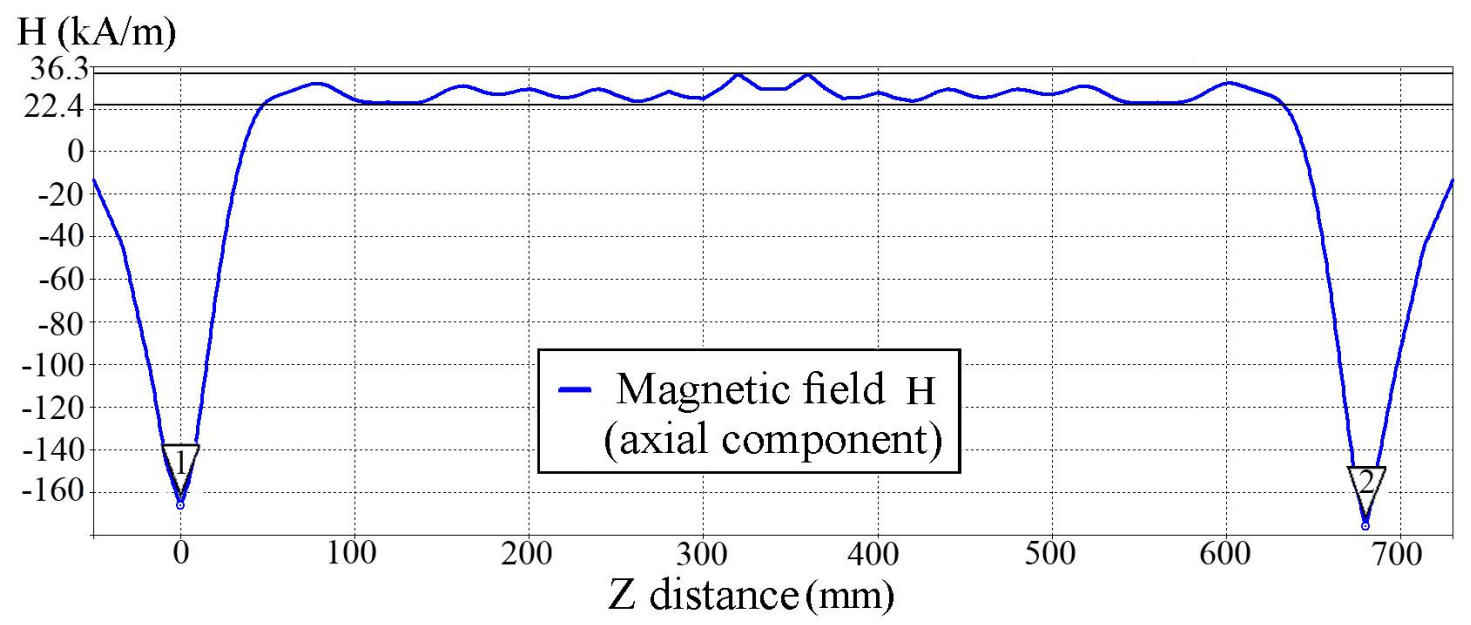

Fig. 18. Magnetic field strength at the center of the rings $(x=0, y=0)$ along the $\mathrm{z}$ axis.

It is possible to notice that for a smaller array of magnets, the uniformity of the field is more evident, due to the reduced size of the structure. As for a larger arrangement, the main difficulty is to control the field variation, since the increase in the size of the structure also increases the complexity for obtaining a uniform field along the structure's axis. It is important to note that the permanent magnet NLTL experiment in [9] produced RF, despite having an axial magnetic field variation of approximately $12.4 \%$ around the average value of $27.65 \mathrm{kA} / \mathrm{m}$ over a $9.25 \mathrm{~cm}$ extension for a 30.0 
Journal of Microwaves, Optoelectronics and Electromagnetic Applications, Vol. 20, No. 3, September 2021

DOI: http://dx.doi.org/10.1590/2179-10742021v20i3254769

cm NLTL structure (using NdFeB magnets) and a variation of approximately $23.7 \%$ around the average value of $59.4 \mathrm{kA} / \mathrm{m}$ over a $7.5 \mathrm{~cm}$ extension for a $30.0 \mathrm{~cm}$ NLTL structure (using ceramic magnets), in this case with much less uniformity for the same NLTL length $(30 \mathrm{~cm})$ than that of the magnetic field obtained in this work. Therefore, we believe that the inversion of the axial magnetic orientation near the ends of the NLTL should not affect the dynamic characteristics of the RF modulation expected at the NLTL output. Besides, RF generation occurs along the line as the pulse current propagates towards the line output.

\section{CONCLUSIONS}

The selection of neodymium permanent magnets using the Ashby method was very efficient for applications in the axial magnetic bias of gyromagnetic NLTLs considering the choice among a database of 574 manufacturer datasheets found from an internet search. Despite having some limitations regarding the maximum operating temperature, neodymium magnets perform very well with a smaller volume of material. This allows for a compact NLTL with high demagnetization and mechanical resistance characteristics. A ring structure composed of neodymium blocks was simulated using the CST Studio Suite software. Despite not having reached an ideal and almost constant uniform field in the region of interest, it was possible nevertheless to obtain a magnetic field with a variation of less than $5.8 \%$ around the average value of $30.5 \mathrm{kA} / \mathrm{m}$ over an $18.5 \mathrm{~cm}$ extension for a NLTL structure of $26.6 \mathrm{~cm}$ length. A variation of less than $23.6 \%$ around the average value of $29.4 \mathrm{kA} / \mathrm{m}$ over a $58.8 \mathrm{~cm}$ extension for a $68.0 \mathrm{~cm}$ NLTL structure was achieved. Therefore, a nearly uniform magnetic field within the NLTL operating range (20-40 kA/m), according to the initial requirements, has been demonstrated in simulations. It is intended that the results presented here are used as a basis for the design and analysis of an experimental structure that is already being assembled in the laboratory, converging to a validation of the results. The next step is to assemble the apparatus to fix the blocks of magnets according to the arrangement design obtained in this work. This paper showed aspects of the use of permanent magnets to replace solenoids in NLTLs for space applications, decreasing the mass and volume of the massive solenoid to produce the bias axial magnetic field as required for the gyromagnetic line operation in space.

\section{ACKNOWLEDGMENT}

The authors thank the National Institute for Space Research - INPE and the Associated Plasma Laboratory - LABAP for providing the facilities for this research. This work was supported in part by CAPES under Grant 88887.492309/2020-00, CNPq under Grant 306540/2019-3, FAPESP under Grant 2018/260862 and SOARD - USAF under Grant FA9550-18-1-0111.

\section{REFERENCES}

[1] J. O. Rossi, F. S. Yamasaki, E. Schamiloglu, J. J. Barroso and U. C. Hasar, "Operation analysis of a novel concept of RF source known as gyromagnetic line", 2017 SBMO/IEEE MTT-S International Microwave and Optoelectronics Conference (IMOC), Aguas de Lindoia, 2017, pp. 1-4, doi: 10.1109/IMOC.2017.8121122. 
[2] L. P. Silva Neto, J. O. Rossi, J. J. Barroso and E. Schamiloglu, "High-Power RF Generation From Nonlinear Transmission Lines With Barium Titanate Ceramic Capacitors”, IEEE Transactions on Plasma Science, vol. 44, no. 12, pp. 3424-3431, Dec. 2016, doi: 10.1109/TPS.2016.2628324.

[3] N. S. Kuek, A. C. Liew, E. Schamiloglu and J. O. Rossi, "Pulsed RF oscillations on a nonlinear capacitive transmission line", IEEE Transactions on Dielectrics and Electrical Insulation, vol. 20, no. 4, pp. 1129-1135, August 2013, doi: 10.1109/TDEI.2013.6571427.

[4] E. G. L. Rangel, J. O. Rossi, J. J. Barroso, F. S. Yamasaki and E. Schamiloglu, "Practical Constraints on Nonlinear Transmission Lines for RF Generation", IEEE Transactions on Plasma Science, vol. 47, no. 1, pp. 1000-1016, Jan. 2019, doi: 10.1109/TPS.2018.2876020.

[5] I. V. Romanchenko, V. V. Rostov, V. P. Gubanov, A. S. Stepchenko, A. V. Gunin, and I. K. Kurkan, "Repetitive subgigawatt rf source based on gyromagnetic nonlinear transmission line", Rev. Sci. Intrum., vol. 83, no. 7, sp.074705. doi: $10.1063 / 1.4738641$.

[6] D. V. Reale, J.-W. B. Bragg, N. R. Gonsalves, J. M. Johnson, A. A. Neuber, J. C. Dickens, and J. J. Mankowski, "Biasfield controlled phasing and power combination of gyromagnetic nonlinear transmission lines", Rev. Sci. Instrum., vol. 85, sp. 054706, May. 2014, doi: 10.1063/1.4878339.

[7] T. L. Gilbert, "A phenomenological theory of damping in ferromagnetic materials", in IEEE Transactions on Magnetics, vol. 40, no. 6, pp. 3443-3449, Nov. 2004, doi: 10.1109/TMAG.2004.836740.

[8] J-W. B. Bragg, J. C. Dickens, and A. A. Neuber, "Material selection considerations for coaxial, ferrimagnetic-based nonlinear transmission lines", J. Appl. Phys., vol. 113, no. 6, p. 064904, Feb. 2013, doi:10.1063/1.4792214.

[9] J. Bragg, J. Dickens and A. Neuber, "Magnetic biasing of ferrite filled nonlinear transmission lines," 2010 IEEE International Power Modulator and High Voltage Conference, Atlanta, GA, USA, 2010, pp. 600-603, doi: 10.1109/IPMHVC.2010.5958430.

[10] F. S. Yamasaki, J. O. Rossi, L. C. Silva, E. G. L. Rangel and E. Schamiloglu, "Operation of a Gyromagnetic Line with Magnetic Axial Bias", 2019 IEEE Pulsed Power \& Plasma Science (PPPS), Orlando, FL, USA, 2019, pp. 1-4, doi: 10.1109/PPPS34859.2019.9009907.

[11] J. O. Rossi, F. S. Yamasaki, E. Schamiloglu and J. J. Barroso, "Analysis of nonlinear gyromagnetic line operation using LLG equation", 2017 IEEE 21st International Conference on Pulsed Power (PPC), Brighton, 2017, pp. 1-3, doi: 10.1109/PPC.2017.8291172.

[12] J. H. Jensen and M. G. Abele, "Generation of highly uniform magnetic fields with magnetized wedges", IEEE Transactions on Magnetics, vol. 34, no. 4, pp. 2316-2323, July 1998, doi: 10.1109/20.703871.

[13] T. Zhang, C. Jiang, Huibin Xu, and M. Jianqin, "Permanent-magnet longitudinal fields for magnetostrictive devices", $J$. Appl. Phys., vol.101, no. 3, p. 034511,Feb. 2017, doi: 10.1063/1.2435809.

[14] G. Aubert, "Cylindrical permanent magnet with longitudinal induced field", U. S. Patent 5.014.032, may. 7, 1991. Available: https://patents.google.com/patent/WO1988002924A1/en, Accessed Feb. 15, 2020.

[15] M. Peters, and C. Leyens, "Aerospace and space materials", Materials Science and Engineering. vol 3, 2009.

[16] K. Fayazbakhsh, and A. Abedian, "Materials selection for applications in space environment considering outgassing phenomenon", Advances in Space Research.vol.45, p. 741-749, 2010, doi https://doi.org/10.1016/j.asr.2009.11.017.

[17] R. D. Karam, "Satellite thermal control for system engineers", Progress in Astronautics and Aeronautics, vol. 181, AIAA, Cambridge, p. 274. 1998.

[18] V.L. Pisacane, "Fundamentals of Space Systems”. Oxford Univ. Press, England, p. 424. 2005

[19] J. -W. Bragg, J. C. Dickens and A. A. Neuber, "Temperature dependence of ferrimagnetic based nonlinear transmission line", 2011 IEEE Pulsed Power Conference, Chicago, IL, USA, 2011, pp. 182-184, doi: 10.1109/PPC.2011.6191411.

[20] J. -W. Bragg, J. C. Dickens and A. A. Neuber, "Ferrimagnetic Nonlinear Transmission Lines as High-Power Microwave Sources", IEEE Transactions on Plasma Science, vol. 41, no. 1, pp. 232-237, Jan. 2013, doi: 10.1109/TPS.2012.2226169.

[21] S. R. Trout and G. D. Wooten, "Selection and specification of permanent magnet materials", Proceedings: Electrical Insulation Conference and Electrical Manufacturing and Coil Winding Technology Conference (Cat. No.03CH37480), Indianapolis, IN, USA, 2003, pp. 59-63, doi: 10.1109/EICEMC.2003.1247855..

[22] CST STUDIO SUITE ${ }^{\circledR}$, CST - Computer Simulation Technology AG, www.cst.com. 